\title{
AN INNOVATIVE ASSAY FOR THE ANALYSIS OF IN VITRO ENDOTHELIAL REMODELING: EXPERIMENTAL AND COMPUTATIONAL EVIDENCE.
}

\section{This is a pre print version of the following article:}

Original Citation:

\section{Availability:}

This version is available http://hdl.handle.net/2318/1572036

since 2017-05-12T18:05:50Z

Published version:

DOI: $10.1002 / j c p .25468$

Terms of use:

Open Access

Anyone can freely access the full text of works made available as "Open Access". Works made available under a Creative Commons license can be used according to the terms and conditions of said license. Use of all other works requires consent of the right holder (author or publisher) if not exempted from copyright protection by the applicable law. 
This is the author's final version of the contribution published as:

Scianna, M; Bassino, E; Munaron, L. AN INNOVATIVE ASSAY FOR THE ANALYSIS OF IN VITRO ENDOTHELIAL REMODELING:

EXPERIMENTAL AND COMPUTATIONAL EVIDENCE.. JOURNAL OF CELLULAR PHYSIOLOGY. None pp: ---.

DOI: $10.1002 /$ jcp. 25468

When citing, please refer to the published version.

Link to this full text:

http://hdl.handle.net/2318/1572036 


\title{
AN INNOVATIVE ASSAY FOR THE ANALYSIS OF IN VITRO ENDOTHELIAL REMODELING: EXPERIMENTAL AND COMPUTATIONAL EVIDENCE.
}

\author{
Scianna Marco ${ }^{1,+}$, Bassino Eleonora ${ }^{2,+}$, Munaron Luca ${ }^{2,3^{*}}$ \\ ${ }^{1}$ Department of Mathematical Sciences, Politecnico di Torino, Corso Duca degli Abruzzi 24, 10129 Torino, Italy, \\ ${ }^{2}$ Department of Life Sciences and Systems Biology, ${ }^{3}$ Centre for Nanostructured Interfaces and Surfaces (NIS), \\ University of Torino, Via Accademia Albertina 13, 10123, Torino, Italy; \\ + these authors equally contributed to this work
}

\author{
*Correspondence to: \\ Luca Munaron, PhD \\ Associate Professor of Physiology \\ Dept. Life Sciences and Systems Biology \\ University of Turin, ITALY \\ Via Accademia Albertina 13 \\ 10123 Torino, ITALY \\ phone ++390116704667 \\ email: luca.munaron@unito.it
}

Key words: angiogenesis, matrix invasion, human microvascular endothelial cells, mathematical model

Running title: A new method to investigate endothelial remodeling in vitro.

Total number of text figures and table: 4 Figures and 2 Tables.

\begin{abstract}
The molecular and cellular mechanisms underlying vascular remodeling are currently investigated several experimental strategies which aim to mimic the complex environmental conditions found in vivo. Some of them focus on the tubulogenic activity of dispersed endothelial cell populations, while others evaluate vascular sprouting. Here we propose a new method to assess matrigel invasion starting from confluent or subconfluent monolayers of human microvascular ECs (HMVEC) seeded on different substrates. The experimental setting is also validated by an improved hybrid multiscale mathematical approach, which integrates a mesoscopic grid-based cellular Potts model, that describes HMVEC phenomenology, with a continuous one, accounting for the kinetics of diffusing growth factors. Both experimental and theoretical approaches show that the endothelial potential to invade, migrate and organize in tubule structures is a function of selected environmental parameters. The present methodology is intended to be simple to use, standardized for rapid screening and suitable for mechanistic studies.
\end{abstract}




\section{Introduction}

Blood vessel formation is a multiscale event that can occur through different mechanisms, including vasculogenesis and sprouting angiogenesis. The overall process is largely mediated by the redundant activity of soluble and fixed environmental factors, respectively including growth factors, such as VEGFs and FGF, and extracellular matrix (ECM) components, such as collagen, fibronectin and gelatin (Munaron 2006; Dister et al., 2003; Munaron et al., 2000). In particular, the dynamic interactions between microvascular endothelial cells (EC) and ECM proteins are mediated by integrins, a superfamily of cell surface receptors whose selective recruitment regulates the angiogenic process. In more details, the complex $\alpha v \beta 3$ has been identified as a marker of angiogenesis (Brook et al., 2015).

Vascular remodeling occurs during development as well as in adult life; however, it can also become pathological, as in the cases of chronic inflammatory diseases, vasculopathies, degenerative disorders, ischemia-associated tissue injury and cancer. Moreover, the control of neovascularization is of great interest in the field of tissue engineering, in order to increase biocompatibility and reabsorption of biomaterials to be employed for a great number of clinical applications (Ahsan et al., 2005; Yarlagadda et al., 2005; Carmeliet 2000). For these reasons, the establishment of easy to use, statistically robust and hopefully high throughput experimental protocols, which can be readily suitable to evaluate the angiogenic potential in vitro by the use of EC cultures, is increasingly required.

In this respect, some approaches, such as the 'scratch wound healing' and transwell-based methods, are mainly focused on EC migration, one of the events required for angiogenesis. On the other hand, other assays that resemble the formation of tubules are regarded as representative of the later stages of angiogenesis (differentiation) and are extensively used to test novel compounds for their pro- or anti-angiogenic activity (Kleinman et al., 1986). In particular, to perform the 'endothelial tube formation assay' (or 'tubulogenesis assay'), EC are initially plated as a dispersed population in a tridimensional matrix, usually Matrigel or similar substrates, which favors cell migration and 
differentiation in the virtual absence of cell proliferation (Benton et al., 2014). Beside its oversimplification, and the lack of a true lumen formation, this experimental protocol revealed very suitable and useful to investigate the molecular mechanisms underlying angiogenic regulation, as shown by the great amount of related publications. However, this approach does not account for sprouting angiogenesis often occurring during neovascularization. During this event EC move from a confluent compact monolayer (the preexisting vessel) to the ECM, that they invade and digest, and eventually organize in tubule structures. Current strategies focused on endothelial sprouting involve tridimensional spheroids of cancer cell-EC co-cultures and aortic ring assays (Benton et al., 2014). Alternative techniques allow to realistically reproduce morphologic steps and molecular events controlling human capillary tube and lumen formation in three-dimensional extracellular matrices (Davis et al., 2000; Koh et al., 2008; Vailhè et al., 2001; Saunders et al., 2006).

However, as far as we know, no experimental protocol has successfully assessed the tubulogenic activity of pure microvascular endothelial populations starting from bidimensional monolayers coated on controlled substrates. This condition is optimal for the formation of interconnected tubules that mimic the in vivo blood vasculature in a highly simplified system in which tubule development is observed over a 4-24 hrs time span. This issue is addressed here by an easy-to-use novel Ibidi-based set up, suitable to quantitatively assess in vitro endothelial sprouting in highly controlled conditions. Selected features of the experimental setting are also reproduced and validated by a proper cellular Potts model-based computational approach. 


\section{Materials and Methods}

\section{Cell culture}

Human microvascular endothelial cell line (HMVEC) was purchased from Lonza (Switzerland), grown in Endothelial Cell Growth Medium (EGM-MV-VEGF) (Lonza, Switzerland) and maintained at $37^{\circ} \mathrm{C}$ in a humidified atmosphere of $5 \% \mathrm{CO}_{2}$.

\section{Cell culture medium and extracellular matrix}

Endothelial Cell Growth Medium (EGM-MV-VEGF) (Lonza, Switzerland) is optimized for the culture of human microvascular endothelial cells; it contains $5 \%$ of serum and VEGF for rapid proliferation. EGM-MV-VEGF used in this work contains the following components: $5 \mathrm{ng} / \mathrm{ml} \mathrm{rh}$ VEGF, $5 \mathrm{ng} / \mathrm{ml} \mathrm{rh}$ EGF, 5ng/ml rh FGF basic, $15 \mathrm{ng} / \mathrm{ml} \mathrm{rh}$ IGF-1, $10 \mathrm{mM}$ L-Glutamine, $1.0 \mu \mathrm{g} / \mathrm{ml}$ Hydrocortisone Hemisuccinate, $0.75 \mathrm{U} / \mathrm{ml}$ Heparin Sulfate, $50 \mu \mathrm{g} / \mathrm{ml}$ Ascorbic Acid.

Dulbecco's Modified Eagle's Medium (DMEM) (Sigma-Aldrich) is a complete liquid form of the high glucose modification of Dulbecco's original formulation that contains four-fold concentrations of the amino acids and vitamins. The DMEM high glucose medium, used in this work, differs from the original formula, because it is, also, supplemented with L-alanyl-L-glutamine dipeptide, $10 \%$ of serum and $10 \mathrm{mM}$ L-Glutamine. Gelatin Solution (Sigma-Aldrich). Gelatin Type B, 2\% in $\mathrm{H}_{2} \mathrm{O}$, is a heterogeneous mixture of water-soluble proteins of high average molecular masses, present in collagen. Proteins are extracted by boiling the relevant skin, tendons, ligaments, bones, etc. in water. Type B is derived from lime-cured tissue. This product was use as a cell culture substratum at $1-5 \mu \mathrm{g} / \mathrm{cm}^{2}$ or $0.5-50 \mu \mathrm{g} / \mathrm{mL}$.

Matrigel (BD Matrigel Membrane Matrix Growth Factor Reduced-GFR). BD Bioscences is suited for applications where a more highly defined basement membrane preparation is desired. Its major component is laminin, followed by collagen IV, heparan sulfate proteoglycans, entactin/nidogen. 
Matrigel contains also TGF-beta, epidermal growth factor, insulin-like growth factor, fibroblast growth factor, tissue plasminogen activator, and other growth factors (Vukicevic et al., 1992; McGuire et al., 1989, see Table 1).

\section{Endothelial tube formation assay (ETFA).}

To assess invasion and angiogenic activity Ibidi culture-inserts (Ibidi, Martinsried, Germany) were employed. The Ibidi culture-Insert is a manufactured from biocompatible silicone material. Cell suspension can be placed in one or both wells allowing to grow cells in the designated areas only (see Table 2). The delimited central area of each well was then coated with Matrigel GFR previously polymerized for $30 \mathrm{~min}$ at $37^{\circ} \mathrm{C}$, see Fig. 1. In our experiments, Matrigel coating simulated extracellular matrix (ECM); Matrigel, indeed, contained not only basement membrane components (collagens, laminin, and proteoglycans) but also matrix degrading enzymes, their inhibitors and growth factors. Subconfluent $\left(10 \times 10^{4}\right.$ cells/well $)$ or confluent $\left(20 \times 10^{4}\right.$ cells/well $)$ HMVECs (SC-HMVECs and C-HMVECs, respectively) were plated in the outer region of each well (i.e., the area without Matrigel coating, see Fig. 1). After 18hrs, the Ibidi device was removed from each well. The subsequent HMVEC invasion, migration and network formation within the Matrigel coated central area were then periodically observed by phase-contrast microscopy using 10x objective magnification (Olympus IX50, equipped with a Cool SNAP-Pro charge-coupled device (CCD) camera), until $72 \mathrm{hrs}$. The time-lapse representative images of the computational assay show different stages of migration, invasion and capillary-like network formation. Experimental images were then processed using ImageJ (National Institutes of Health, NIH, US) software.

\section{Quantification of HMVEC invasion and tubulogenic activity}

Endothelial tube formation was analyzed by the use of Angiogenesis Analyzer plugin of ImageJ software. This tool allows to easily quantify the ETFA experiment images by extracting 
characteristic information of the network it allow the network analysis organization of individual ECs images taken in phase contrast or in fluorescence. Tubulogenic potential is expressed as a set of parameters including the total tube length $(\mu \mathrm{m})$ and the total number of junctions.

\section{Viability assay}

SC-HMVECs were seeded in the outer region of each well. After $72 \mathrm{hrs}$ cell viability was evaluated by the CellTiter 96® AQueous Non-Radioactive Cell Proliferation Assay (Promega, Madison, WI, USA), using 3-(4,5-dimethylthiazol-2-yl)-5-(3-carboxymethoxyphenyl)-2-(4-sulfophenyl)-2H tetrazolium, inner salt (MTS). MTS conversion into the aqueous soluble formazan product is accomplished by dehydrogenase enzymes found in metabolically active cells. Formazan product was measured with a FilterMax F5 Microplate reader (Molecular Devices, US) at $490 \mathrm{~nm}$, as absorbance is directly proportional to the number of viable cells.

\section{Statistics}

Statistical significance of all experiments was evaluated by Kaleidagraph software (Synergy Software, USA) using nonparametric Wilcoxon test. The Wilcoxon test was used for cell tubulogenesis analysis because the percentages of tubulogenesis were not normally distributed. All values are presented as the mean \pm standard error (SE). For each experimental condition (CHMVEC, SC-HMVEC both grown in DMEM 10\% or EGM-MV-VEGF) five technical replicates were performed; three biological replicates were done for each experiment: $\mathrm{N}=3$. Results with $p$ values $<0.05$ were considered statistically significant.

\section{Mathematical model}

The experimental assay was reproduced with a hybrid multilevel approach, able to span the multiple scales underlying HMVEC invasion and tubule organization. In particular, at the cellular level, an extended version of the cellular Potts Model (CPM), a lattice-based Monte Carlo technique which 
follows an energy minimization principle, was the core of the simulation system and described the phenomenology of HMVEC population, capturing the mechanisms of cell migration, polarization, adhesion, and proliferation. At the molecular level, a continuous model reproduced instead the kinetics and the morphogenic activity of chemical growth factors, dealing with their production, diffusion and degradation. The two submodels were then interfaced and integrated in order to link the behavior of cells to their biochemical state. In this respect, the multicellular invasion across the matrix, and the subsequent network formation, emerged as a consequence of cellular and subcellular dynamics. The statistical analysis of the model outcomes was performed by MatLab (The MathWorks Inc., US) procedures, specifically encoded for this work. In particular, coherently with the experimental protocol, we focused on network determinants such as the total tubule length/area and the number of junctions. All values are presented as the mean \pm standard error (SE) over 50 independent simulations. For a detailed description of the mathematical approach see the Supplementary Information. 


\section{Results}

The experimental assay described in this work was set up to measure the ability of a human microvascular endothelial monolayer to invade the matrix and self-organize in capillary-like networks (Fig. 1). In particular, to study the collective cell dynamics involved in the overall process, we considered HMVEC in different experimental growth conditions, that differed for three environmental parameters critically involved in neovascularization: cell-cell contact (confluent or subconfluent monolayers), growth factor enrichment (DMEM 10\% or EGM-MV-VEGF medium), and cell adhesion to the substrate (presence or absence of gelatin).

When grown in both EGM-MV-VEGF or in DMEM 10\% media and plated in the absence of any coating substrate, SC-HMVEC started invading the Matrigel with a typical 'wound healing-like' behavior (Fig. 2A). In both cases, after nearly $18 \mathrm{hrs,} \mathrm{the} \mathrm{cells} \mathrm{starting} \mathrm{from} \mathrm{the} \mathrm{front} \mathrm{of} \mathrm{the}$ monolayer drafted to organize in tubule structures. Later ( $72 \mathrm{hrs})$, an extended area of Matrigel was covered by tubule structures: in particular the total vessel length and total number of junctions were highly dependent on the growth factor enrichment of the medium, being drastically more pronounced in EGM-MV-VEGF than in DMEM 10\% (Fig. 2A-B). The same phenomenology, with very similar dynamics, was captured by the computational approach (Fig. 3A-B), although the resulting patterns were characterized (in average) by longer branches and fewer junctions.

Interestingly, the invasive behavior of SC-HMVECs growing on gelatin-coated substrate drastically differed depending on the medium (Fig. 2D). In particular, HMVEC grown in EGM-MV-VEGF displayed limited Matrigel invasion without tubule organization (Fig. 2D1), although cell viability was unaltered (Supplementary Fig.1, panel A). The already poor tubulogenic potential of HMVEC cultured in DMEM 10\% medium was instead not significantly affected by gelatin coating, in terms of both total vessel length and total number of junctions (Fig. 2D2). The mathematical approach substantially confirmed these data (Fig. 3A-B).

C-HMVEC resulted viable only when grown in EGM-MV-VEGF, while the viability in DMEM $10 \%$ was too low (less than $46 \%$ ) to allow any suitable and statistically robust experiments. C- 
HMVEC grown in EGM-MV-VEGF were initially closely packed each other (24 hrs). As a consequence, in an early phase they invaded Matrigel in a 'wound healing-like' fashion more prominently than SC-HMVEC: a leading front of the population advanced in fact more significantly and compactly across the Matrigel (Fig. 2C1). Conversely, in later phase (72 hrs) the tubulogenic potential of C-HMVEC appeared drastically reduced if compared to SC-HMVEC, in terms of both total vessel length and total number of junctions (Fig. 2A-F). The corresponding sets of mathematical simulations captured the same behavior (Fig. 3A-C); in particular, the emerging in silico tubule structures were more poorly extended and typically formed by shorter and thicker branches.

Similarly to the case of SC-HMVECs, the tubulogenic potential of C-HMVEC grown in EGM-MVVEGF was further drastically prevented when the monolayer was plated on a gelatin-coated substrate (Fig. 2D3-E). These evidences were nicely reproduced by mathematical simulations as well (Fig. 3A-C). 


\section{Discussion}

Here we present a novel assay which aims to provide an in vitro experimental tool to investigate the role of environmental parameters on the formation of endothelial tubule network starting from a simple monolayer of cells growing on a selected adhesion substrate.

The experimental evidence was further validated with a proper theoretical framework. In particular, the proposed mathematical approach is firmly anchored in the computational literature, as it is essentially based on a series of Cellular Potts Models dealing with different scenarios of vascular progression. However, when compared to the previous published works, it presents some relevant novelties, which were introduced to better reproduce the specific experimental conditions.

A preliminary consideration concerns the relevance of culture medium. Both in vitro and in silico evidences clearly indicate that the maximal morphogenic potential can be observed in HMVEC grown in EGM-MV-VEGF, a medium enriched with proangiogenic factors, while tubulogenesis is significantly reduced in DMEM 10\% (Figs. 2 and 3). This observation is expected according to the conventional tubulogenesis assays described in literature for micro and macrovascular endothelium (Benton et al., 2014).

On the other hand, the role played by cell confluence is not trivial. HMVEC starting from monolayer at sub-confluence density show an enhanced ability to form mature tubule networks when compared with confluent populations. This evidence suggests that the cell density of the monolayer is inversely proportional to the tubulogenic potential. Interestingly, this correlation is unlikely to be due to an overall decrement in cell migration expected for the enhanced cell-cell interactions at confluence: the initial 'wound-like' compact invasion of Matrigel at 24 hours is in fact more prominent starting from C-HMVEC than from SC-HMVEC. The increment in the intercellular adhesive interactions (consequent to the higher cell density) partially disrupts the regulation of the chemotactic component of cell migration, which is required for a proper networking behavior. This mechanism, called 'contact inhibition of chemotaxis', has been widely 
recognized and described in the experimental and computational literature (Hellstrom et al., 2007; Lin et al., 2015; Scianna et al., 2015).

In this respect, our method will be well employed for future investigations aimed to shed light on this particular issue: indeed, differently from other approaches, it is suitable for time lapse fluorescence microscopy imaging, aimed at measuring the dynamics of intracellular messengers critically involved in endothelial migration and organization in tubule networks. Among them, calcium signaling calcium-related machinery have been widely recognized as key mediators of specific steps occurring during angiogenic events (Munaron, 2012). In particular, previous reports from our group established a correlation between tubulogenic potential and calcium signals in patophysiological conditions (Fiorio Pla et al., 2008; Munaron, 2012).

From the computational point of view, calcium dynamics should be approached with continuous methods (i.e., reaction-diffusion equations) based on experimental data mainly obtained from electrophysiological and fluorimetric measurements. In particular, the impact of cell confluence on the calcium machinery could be accounted in the reaction term of the corresponding equation. Such new mathematical ingredients should be then interfaced with the lattice-based CPM, as previously shown by our group in previous reports (Scianna et al., 2011; Munaron et al., 2012).

In particular, the resulting mathematical approach will be able to systematically characterize the relationship between intracellular calcium dynamics, cell confluence and the resulting pattern. For instance, a promising study could be focused on the modeling counterparts of biomedical strategies that have the potential to disrupt network formation, in the case of pathological situations. This way, it would be possible not only to confirm the efficacy of some currently available anti-angiogenic therapies but also to suggest novel and experimentally testable treatments. Since the interaction with the substrate is critical for endothelial cell adhesion, migration, signaling and angiogenic potential (Muhammad et al., 2007; Shen et al., 2011; Neve et al, 2014), we decided to compare endothelial remodeling starting from cell monolayers plated with or without gelatin, widely used to enhance cell attachment. We show that gelatin completely prevents tubulogenesis of both C-HMVEC and 
SC-HMVEC cultured in EGM-MV-VEGF, but not in DMEM 10\% (Fig. 2). These data suggest that the proangiogenic potential of factors contained in EGM-MV-VEGF (mainly VEGF and FGF) could be at least partially prevented by gelatin, leading to a relevant anti-tubulogenic effect. This activity is substantially independent on cell confluence and, thus, on the previously-cited physical constraints related to cell-cell contact (see Fig. 2). We can exclude a significant contribution of putative increased mitogenic potential in EGM-MV-VEGF/gelatin condition, that could eventually reduce migration and differentiation, since gelatin coating does not affect HMVEC proliferation induced by EGM-MV-VEGF (Supplementary Fig.1 panel A). Our results with gelatin-coating are in nice agreement with several reports showing that differences in the degree of cell-ECM adhesion could promote distinct endothelial cell behaviors important for angiogenesis (Neve et al., 2014). Gelatin is largely used to enhance endothelial spreading and adhesion on its substrate and these events regulate the expression of genes associated with angiogenesis. In particular, VEGF-related signaling and gene expression result highly sensitive to cell adhesion: among the genes most highly upregulated in un-spread cells in the presence of VEGF are those included in the microarrays of angiogenesis that involve endothelial invasion, migration and organization (VEGF and Angiopoietin receptors, MMP14 and some integrins) (Shen et al, 2011; Bell et al., 2001; Gerritsen et al., 2002). Taken together, these (and other) observations suggest that limiting cell adhesion through multiple mechanisms, whether via changes in ligand density or cell spreading, induced the expression of genes associated with the invasive processes of angiogenesis. Further mechanistic studies should deserve attention on this topic, and the method described in this paper could usefully contribute. The present Ibidi-based assay does not aim at substituting the well-established and powerful alternative techniques already used to study the endothelial remodeling in vitro. Nevertheless, it may provide easy to use and standardized additional information on the mechanisms underlying the morphogenic behavior of vascular endothelium. 


\section{Acknowledgements}

M.S. performed the research, analysed the data and wrote the paper.

E.B. performed the research, analysed the data and wrote the paper.

L.M. designed the research study, analysed the data, wrote the paper and contributed essential reagents or tools. 


\section{References}

Ahsan T, Nerem RM. 2005. Bioengineered tissues: the science, the technology, and the industry. Orthod Craniofac Res 8:134-40.

Bell SE, Mavila A, Salazar R, Bayless KJ, Kanagala S, Maxwell SA, Davis GE. 2001. Differential gene expression during capillary morphogenesis in 3D collagen matrices: regulated expression of genes involved in basement membrane matrix assembly, cell cycle progression, cellular differentiation and G-protein signaling. J Cell Sci. 114:275573.

Benton G, Arnaoutova I, George J, Kleinman HK, Koblinski J. 2014. Matrigel: From discovery and ECM mimicry to assays and models for cancer research. Advanced Drug Delivery 79:3-18.

Brook PC, Montgomery AM, Rosenfeld M, Reisfeld RA, Hu T, Klier G, Cheresh DA. 2015. Integrin alpha $\mathrm{v}$ beta 3 antagonists promote tumor regression by inducing apoptosis of angiogenic blood vessels Cell 79:1157-1164.

Carmeliet P. 2000. Mechanisms of angiogenesis and arteriogenesis. Nat Med 6:389-95.

Davis GE, Black SM, Bayless KJ. 2000. Capillary Morphogenesis During Human Endothelial Cell Invasion Of Three-Dimensional Collagen Matrices. 2000. In Vitro Cell. Dev. Biol. Animal 36:513-519.

Distler JW, Hirth A, Stolarska MK, Gay RE, Gay S, Distler O. 2003. Angiogenic and angiostatic factors in the molecular control of angiogenesis. Q J Nucl Med 47:149-161.

Fiorio Pla A, Grange C, Antoniotti S, Tomatis C, Merlino A, Bussolati B, Munaron L. 2008. Arachidonic acid-induced $\mathrm{Ca} 2+$ entry is involved in early steps of tumor angiogenesis. Mol Cancer Res 6:535-45.

Gerritsen ME, Soriano R, Yang S, Ingle G, Zlot C, Toy K, Winer J, Draksharapu A, Peale F, $\mathrm{Wu}$ TD, Williams PM. 2002. In silico data filtering to identify new angiogenesis targets from a large in vitro gene profiling data set. Physiol Genomics 10:13-20.

Hellström M, Phng LK, Hofmann JJ, Wallgard E, Coultas L, Lindblom P, Alva J, Nilsson AK, Karlsson L, Gaiano N, Yoon K, Rossant J, Iruela-Arispe ML, Kalén M, Gerhardt H, Betsholtz C. 2007. D114 signalling through Notch1 regulates formation of tip cells during angiogenesis. Nature 445:776-80.

Kleinman HK, McGarvey ML, Hassel JR, Star VL, Cannon FB, Laurie GV, Martin GR. 1986. Basement membrane complexes with biological activity. Biochemistry 25:312-318.

Koh W, Stratman AN, Sacharidou A, Davis GE. 2008. In Vitro Three Dimensional Collagen Matrix Models of Endothelial Lumen Formation During Vasculogenesis and Angiogenesis. Methods in Enzymology, Vol. 443.

Lin B, Yin T, Wu YI, Inoue T, Levchenko A. 2015. Interplay between chemotaxis and contact inhibition of locomotion determines exploratory cell migration. Nat Commun. 6:6619. 
McGuire PG, Seeds NW. 1989. The interaction of plasminogen activator with a reconstituted basement membrane matrix and extracellular macromolecules produced by cultured epithelial cells. J. Cell. Biochem 40:215-227.

Muhammad H. Zaman. 2007. Understanding the Molecular Basis for Differential Binding of Integrins to Collagen and Gelatin. Biophys J 92:17-9.

Munaron L, Fiorio Pla A. 2000. Endothelial calcium machinery and angiogenesis: Understanding physiology to interfere with pathology. Current Medicinal Chemistry 16:4691-4703.

Munaron L, Scianna M. 2012. Multilevel complexity of calcium signaling: Modeling angiogenesis. World J Biol Chem. 26:121-6.

Munaron L. 2006. Intracellular calcium endothelial cells and angiogenesis. Recent Patents Anticancer Drug Discov 1:105-119.

Munaron L. 2012. Intracellular calcium signaling: holding the balance between health and disease. Curr Med Chem.19:5765-7.

Neve A, Cantatore FP, Maruotti N, Corrado A, Ribatti D. 2014. Extracellular matrix modulates angiogenesis in physiological and pathological conditions. Biomed Res Int. 2014:756078.

Saunders WB, Bohnsack BL, Faske JB, Anthis NJ, Bayless KJ, Hirschi KK and Davis GE. 2006. Coregulation of vascular tube stabilization by endothelial cell TIMP-2 and pericyte TIMP-3. J. Cell. Biol 175:179-191.

Scianna M, Bassino E, Munaron L. 2015. A cellular Potts model analyzing differentiated cell behavior during in vivo vascularization of a hypoxic tissue. Comput Biol Med 63:143-56.

Scianna M, Munaron L, Preziosi L. 2011. A multiscale hybrid approach for vasculogenesis and related potential blocking therapies. Prog Biophys Mol Biol. 106:450-62.

Shen CJ, Raghavan S, Xu Z, Baranski JD, Yu X, Wozniak MA, Miller JS, Gupta M, Buckbinder L, Chen CS. 2011. Decreased Cell Adhesion Promotes Angiogenesis in a Pyk2-dependent Manner. Exp Cell Res. 317:1860-1871.

Vailhé B, Vittet D, Feige JJ. 2001. In vitro models of vasculogenesis and angiogenesis. Lab Invest. $81: 439-52$.

Vukicevic S. Vukicevic I, Kleinman HK Luyten FP, Roberts AB, Rochel NS, Reddi AH. 1992. Identification of multiple active growth factors in basement membrane Matrigel suggests caution in interpretation of cellular activity related to extracellular activity related to extracellular matrix components. Exp Cell Res 202:1-8.

Yarlagadda PK, Chandrasekharan M, Shyan JY. 2005. Recent advances and current developments in tissue scaffolding. Biomed Mater Eng 15:159-77. 


\section{Figure legends}

Figure 1: Experimental setup (see Methods). In each well an Ibidi insert delimited the outer cell culture area and the central Matrigel coated area (see the white dashed lines in the experimental panels). After removing the Ibidi barrier, HMVEC were allowed to invade and migrate within the central Matrigel coated region until $72 \mathrm{hrs}$ (see the corresponding white dashed lines in the experimental panels). The computational domain was setup to closely resemble the in vitro configuration (refer also to Supplementary Fig.1).

Figure 2: Experimental evaluation of HMVEC invasion and pseudo-capillary network formation. A-D. Representative images from HMVEC cultured in different conditions described in the results. A. Initial 'wound-like' phase of Matrigel invasion by subconfluent (SC) HMVEC grown in DMEM $10 \%$ FCS (1) or in EGM-MV-VEGF (2) (12 hrs).

B. Late Matrigel invasion (72 hrs) of HMVEC plated at subconfluence (SC) in DMEM 10\% FCS (1) or in EGM-MV-VEGF (2). Images in 1' and 2' are representative fields taken for tubulogenesis analysis and images in 1" and 2" are an example of the graphical output generated by Angiogenesis Analyzer (see Methods).

C. Early and late Matrigel invasion of HMVEC grown at confluence in EGM-MV-VEGF. Image in 1 ' is representative for the early 'wound-like' migration and images 2 ' and 2" show tubulogenesis at 72 hrs. In particular, image in 2" is an example of the graphical output generated by Angiogenesis Analyzer (see Methods).

D. Late Matrigel invasion (72 hrs) of HMVEC grown at subconfluence (SC) or confluence (C) in DMEM 10\% FCS or in EGM-MV-VEGF and plated on gelatin-coated substrate. Image in 2' is a representative field taken for tubulogenesis analysis and image 2" is an example of the graphical output generated by Angiogenesis Analyzer (see Methods). In A-D, the black lines indicate the position of the Ibidi, delimiting the initial cell plated area from the Matrigel coated region.

E. Plot showing the statistics of tubule length in the experimental conditions of A-D ( $72 \mathrm{hrs})$.

F. Plot showing the statistics of total number of junctions in the experimental conditions of A-D.

In E-F, the values in the plots are given as mean \pm standard error (SE) $(n=3$ independent experiments).

Figure 3. Computational evaluation of HMVEC invasion and pseudo-capillary network formation.

A. Representative simulation images from HMVEC cultured in the different conditions described in the results at the final observation time (72 hrs).

B. Plots showing the statistics of tubule length $(\mu \mathrm{m})$. 
C. Plots showing the statistics of the total number of junctions corresponding to the experimental conditions of A.

Values are given as mean \pm standard error (SE) out of over 50 independent simulations. 